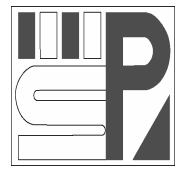

Science Press
Journal of Arid Land

2011, 3(4): 261-267

doi: 10.3724/SP.J.1227.2011.00261

jal.xjegi.com; www.chinasciencejournal.com

\title{
Anatomical and morphological characteristics of Populus euphratica in the lower reaches of Tarim River under extreme drought environment
}

\author{
Li ZHUANG ${ }^{1}$, YaNing CHEN ${ }^{2 *}$, WeiHong $\mathrm{LI}^{2}$, ZhongKe WANG ${ }^{1}$ \\ ${ }^{1}$ College of Biology, Shihezi University, Shihezi 830002, China; \\ ${ }^{2}$ Xinjiang Institute of Ecology and Geography, Chinese Academy of Sciences, Urumqi 830011, China
}

\begin{abstract}
Populus euphratica Oliv. is an old desert tree species that has been naturalized and invades zones along the watercourses in many arid and semiarid regions. The plant species developed some plasticity to adapt to the gradual environmental gradients. The aim of this study was to test the hypothesis that the changes in leaf morphology of $P$. euphratica reflect the adaptability of the plant to the unique environment of the lower reaches of Tarim River in China. The foliar architecture, blade epidermal and internal anatomies of $P$. euphratica were analyzed at different sites along the Tarim River. Compared with the abaxial surface of the leaves, their adaxial surface has more hairs, a greater stomatal density and opening, higher mesophyll proportion, and increased blade thickness, palisade width, and epidermal thickness. The long trichome of the roots found at site 6 in the Yinsu section may be an adapted structure of the plants in arid areas. The mature leaves of $P$. euphratica have comparatively more epidermis and cuticles, well developed palisades and more chloroplasts at different sites compared to the young leaves. Foliar morphological and anatomical variability in $P$. euphratica may be considered an adaptive advantage that enables leaves to develop and function in different habitats, marked by strong variations in solar radiation, air temperature, humidity and water table.
\end{abstract}

Keywords: $P$. euphratica; ecological adaptation; leaf morphology; water stress; Tarim River

In order to survive in different environments, the morphological traits of widely distributed plant species often vary considerably. Leaves are exposed to aerial conditions more than any other plant organs, and the changes in their characters have been interpreted as adaptations to specific environments (Leymarie et al., 1999; Charles et al., 2003). The variations in the morphological and anatomical features of leaves and with the relationships of the amount of sun exposure, the degree of salinity, or water availability have been reported for many species ( $\mathrm{Gu}$ et al., 1999; Maria, 2000; Shin, 2000).

Populus euphratica Oliv. is widely distributed in the desert regions of Central Asia, and developed along the watercourse in the Tarim River valleys of China. The species is known for its ability to grow in a wide range of environmental conditions, such as arid, high salinity, or windy and dusty abominably environments. The area of $P$. euphratica woodlands in the Tarim River basin accounts for $54 \%$ of the species in the world (Zhang et al., 1996; Wang et al., 1999; Li et al., 2006; Wang et al., 2009; Zhuang et al., 2010). The researches of the capacity for $P$. euphratica to establish and grow in extreme environments might favor its recovery and spread.

The size and shape of $P$. euphratica leaves varies at different times in the growing season, which is beneficial for the evaluation of the environmental heterogeneity in the lower reaches of Tarim River. The anatomical and morphological characters of $P$. euphratica leaves were studied in order to analyze the relationship between the external environmental factors and the observed

Received 2011-03-29; accepted 2011-07-06

*Corresponding author: YaNing CHEN (E-mail: Chenyn@ms.xjb.ac.cn.) 
characteristic differences. Therefore, the hypothesis that the anatomical and morphological features of $P$. euphratica leaves are developed in response to the environmental spatial heterogeneity, especially to the changes of water table in extremely arid regions.

\section{Materials and methods}

\subsection{Plant material and growth conditions}

In 2008, $P$. euphratica leaf samples were collected from the following sections: Yahepu $(836 \mathrm{~m}$ a.s.1., $\left.40^{\circ} 29.516^{\prime} \mathrm{N}, 87^{\circ} 50.172^{\prime} \mathrm{E}\right)$, Yingsu (832 $\mathrm{m}$ a.s.1., $\left.40^{\circ} 29.208^{\prime} \mathrm{N}, 87^{\circ} 56.337^{\prime} \mathrm{E}\right)$, Abudali (828 $\mathrm{m}$ a.s.l., $\left.40^{\circ} 24.879^{\prime} \mathrm{N}, 88^{\circ} 03.003^{\prime} \mathrm{E}\right)$, Kardayi (829 m a.s.l., $\left.40^{\circ} 22.339^{\prime} \mathrm{N}, \quad 88^{\circ} 10.243^{\prime} \mathrm{E}\right), \quad$ Alagan $(817 \mathrm{~m}$ a.s.l., $\left.40^{\circ} 08.769^{\prime} \mathrm{N}, 88^{\circ} 21.575^{\prime} \mathrm{E}\right)$, Yiganbjima (811 $\mathrm{m}$ a.s.l., $39^{\circ} 47.288^{\prime} \mathrm{N}, 88^{\circ} 22.671^{\prime} \mathrm{E}$ ), and Kaogan (926 m a.s.1., $\left.41^{\circ} 19.722^{\prime} \mathrm{N}, 88^{\circ} 25.241^{\prime} \mathrm{E}\right)$, which are all located along the Tarim River (Fig. 1). This species has been important to the conservation of the Tarim River for the last 30 years. Five or six plots were established in $50 \mathrm{~m}, 100 \mathrm{~m}, 200 \mathrm{~m}, 300 \mathrm{~m}, 400 \mathrm{~m}$ and $500 \mathrm{~m}$ distances from the river in accordance to the position of the groundwater monitoring wells in each section. The climate in the lower reaches of the Tarim River belongs to the continental warm temperature zone, with dry weather. The average temperature during the coldest month (January) is $-15^{\circ} \mathrm{C}$, and the hottest month (July) is $25^{\circ} \mathrm{C}$. The annual pan evaporation is 2,671.4-2,902.2 $\mathrm{mm}$, with a negative water balance throughout the year. The annual precipitation is 17.4-42.0 mm, most occurring from April to September. The average annual solar radiation is 1,740
$\mathrm{kW} \cdot \mathrm{h} /\left(\mathrm{m}^{2} \cdot \mathrm{a}\right)$, with about $3,000 \mathrm{~h}$ of sunlight. The landform is a composite mode with barchan chains, and longitudinal and shrub dunes.

\subsection{Methods}

The air temperature, humidity and soil moisture were recorded using SAP FLOW RELATIVE RATE SENSORS (PHYECH, plant physiology and ecology monitoring system, Israel). The soil water content was measured by the gravimetric method. The groundwater data was monitored monthly using universal meter. The salinity was measured monthly by in-situ soil salinity. Leaf water potential was measured by HR-33T (Dew-point water potential meter, WESCOR Company, Logan, UT, USA).

In each plot, fully expanded and undamaged leaves of $P$. euphratica from 2.5-3 m height were collected in all directions.

Leaf water content (LWC) during the growing period was measured by collecting $100 \mathrm{~g}$ of leaf material in each direction of three trees. The fresh weight was determined, and then the plant tissues were dried for $48 \mathrm{~h}$ at $60^{\circ} \mathrm{C}$ to determine the dry weight.

The leaf material for the scanning electron microscopy (SEM) was fixed by formal-acetic acid-alcohol (FAA). Samples were dehydrated using increasing strengths of $75 \%$ alcohol and cleared using an ultrasonic wave for $10 \mathrm{~min}$. The samples were then frozen in liquid nitrogen and coated with a film of gold and a sputter coater SCT620. Both the epidermis and the cross-section of each sample were examined under a scanning electron microscope (LEO1430VP SEM), respectively. Foliar architecture was observed using

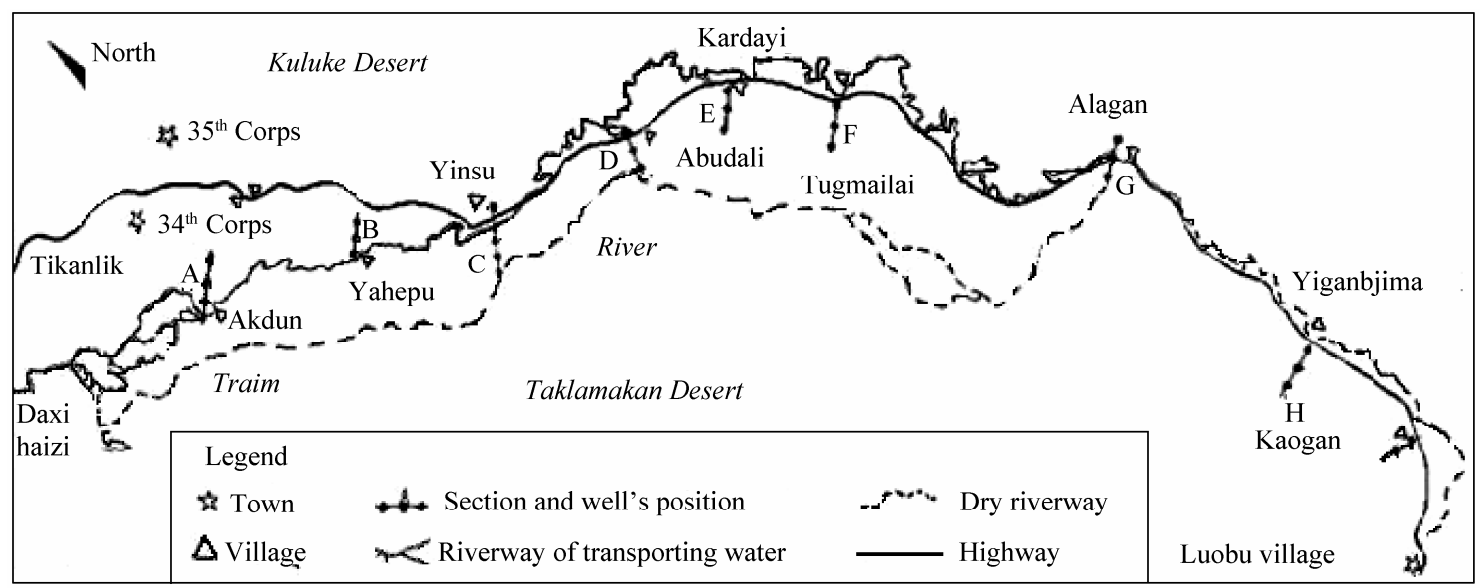

Fig. 1 The sketch of investigation sections in the lower reaches of Tarim River 
transmission election microscopy (TEM). Cleared leaves were fixed in FAA, rinsed thoroughly in a phosphate buffer, then fixed with osmic acid and rinsed with a phosphate buffer again. The samples were dehydrated in increasing strengths of alcohol from 30\% (15 min), 50\% (15 min), 70\% (12 h), 85\% (15 min), 95\% (15 $\mathrm{min})$, to $100 \%$ (15 min, twice). After a series of alcohol-acetone to pure acetone wash and acetone-epoxy resin to pure epoxy resin, the samples were embedded with an epoxy resin and baked in oven from $35-60^{\circ} \mathrm{C}$ (in $5^{\circ} \mathrm{C}$ intervals), after which the samples were cut using a microtome. The microtome sections were stained by uranium acetate and citric acid lead. The cellular structure of each sample was examined using Hitachi H-600X TEM (Tokyo, Japan).

\subsection{Data analysis}

The statistical analysis was performed with SAS software (version 6.12, SAS Institute Inc., Cary, USA). All data were subject to a one-way analysis of variance (ANOVA) and the means were separated by least significant difference (LSD) test at 0.05 level.

\section{Results}

The results of monitoring environmental factors during the period of 2006-2008 in the study area are listed in Table 1 . Soil salinity values reached the highest at $5 \mathrm{~cm}$ depth, up to a maximum of $8.73 \mathrm{~g} / \mathrm{mol}$ at the site 4 of the Yinsu section (Y4) in August 2006. The air temperature in Yinsu section of the lower reaches of Tarim River reached a maximum of $42^{\circ} \mathrm{C}$ in July 2007. The air humidity at the Y1 sampling site reached a minimum of $10 \%$ and, at the $\mathrm{Y} 3$ site attained a maximum of $100 \%$. The measured soil moisture values were from zero to the maximum of $69.2 \%$.

The average annual water table in the five sampling sites of the Yinsu section is shown in Fig. 2. During spring and summer, the water table decreased corresponding to the increase in the distance from the river course in 2006, 2007 and 2008, except in the Y2 site in April 2006 and April 2008. Water tables of the Y1-Y5 sites ranged from $2.55 \mathrm{~m}$ to $6.49 \mathrm{~m}$, and $50 \%$ of them were over $5 \mathrm{~m}$.

The average dry weight of $P$. euphratica leaves is shown in Table 2. A total of 50 leaves of three plants in each direction were collected and measured during August of 2006. The average dry weights of $P$. euphratica leaves at the sites 1 of every section were higher than other sites except Yinsu section where the highest and the lowest values were $1.024 \mathrm{~g}$ and 0.1367 $\mathrm{g}$ in the site 2 and site 4 , respectively.

Daily water potentials of the $P$. euphratica leaves were found to be higher in the morning and evening while the values were lower at noon. The highest value of the water potential was measured in the leaves of the species that occurred $300 \mathrm{~m}$ away from the river (Table 3 ). The average relative water capacity measured in the P. euphratica trees in the Yinsu section are shown in Fig. 3. The Y6 site had the highest average relative water capacity, with an increase of $61.62 \%$.

The leaves of $P$. euphratica are deciduous and become golden yellow color in autumn before senescing. New leaves grow during early spring. Most of the leaves of a healthy P. euphratica tree may wither under extremely environments, such as water stress. When the environment is too harsh, only a small cluster of leaves can survive, along with fewer flowers and fruits. Fully expanded leaves are simple and symmetrical, with an entire margin; however, $P$. euphratica leaves can differ based on their location in the tree, or based on the different growing periods of the plant species. Most of the upper leaves of a mature tree are

Table 1 Environmental factors of Yinsu section during the growing period of $P$. euphretica

\begin{tabular}{|c|c|c|c|c|c|c|c|c|c|c|c|c|c|}
\hline \multirow{3}{*}{ Site } & \multicolumn{4}{|c|}{ Soil depth $(\mathrm{cm})$} & \multicolumn{3}{|c|}{ Air temperature $\left({ }^{\circ} \mathrm{C}\right)$} & \multicolumn{3}{|c|}{ Air humidity (\%) } & \multicolumn{3}{|c|}{ Soil moisture (\%) } \\
\hline & 5 & 10 & 20 & 40 & \multirow{2}{*}{ Max. } & \multirow{2}{*}{ Min. } & \multirow{2}{*}{ Mean } & \multirow{2}{*}{ Max. } & \multirow{2}{*}{ Min. } & \multirow{2}{*}{ Mean } & \multirow{2}{*}{ Max. } & \multirow{2}{*}{ Min. } & \multirow{2}{*}{ Mean } \\
\hline & & Soil s & $/ \mathrm{mol})$ & & & & & & & & & & \\
\hline $\mathrm{Y} 1$ & 6.40 & 6.09 & 3.57 & 4.97 & 42.0 & 20.0 & 31.43 & 62 & 12 & 26.80 & 11.1 & 4.6 & 9.69 \\
\hline $\mathrm{Y} 2$ & 7.41 & 3.50 & 3.55 & 4.09 & 40.5 & 22.5 & 31.76 & 54 & 10 & 20.95 & 15.6 & 6.1 & 12.68 \\
\hline $\mathrm{Y} 3$ & 3.62 & 2.34 & 4.89 & 1.23 & 34.0 & 13.5 & 22.82 & 100 & 26 & 71.93 & 69.2 & 0 & 49.39 \\
\hline Y4 & 8.73 & 7.49 & 5.31 & 2.11 & 41.5 & 18.0 & 31.48 & 54 & 10 & 21.93 & 15.2 & 4.6 & 8.70 \\
\hline Y5 & 7.11 & 2.49 & 3.15 & 1.84 & 38.5 & 20.5 & 30.74 & 56 & 16 & 30.09 & 16.3 & 4.6 & 15.63 \\
\hline
\end{tabular}




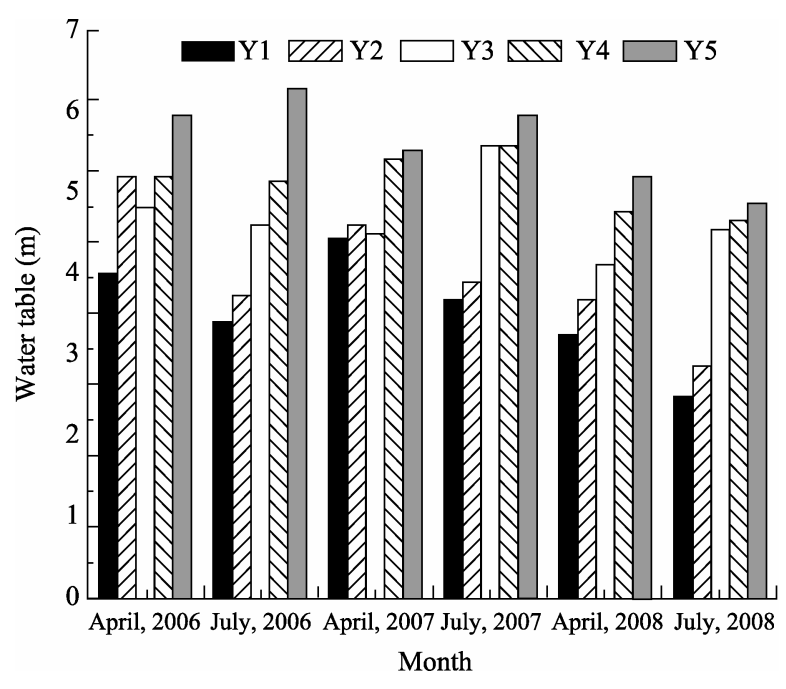

Fig. 2 Mean water tables $(m)$ in Yinsu section during the period of April, 2006 to August, 2008 ovate with an entire margin or vandyke, and the sizes and shapes are very different, ranging from oblong to ellipse. Lanceolate leaves occur on the base of the stem. Therefore, the tree was again named as "Populus with heteromorphous leaves". The texture of the lamina is leathered, but the color varies, with dark green adaxial surfaces and the abaxial surfaces having a woolly appearance and being lighter color, a feature that is especially evident in mature leaves.

The leaves are bifacial, with a biseriate palisade in most sites, but a third, poorly organized stratum was observed in the Y4 site (Fig. 4a). Palisade cells have cylindrical and spongy tissue, arranged regularly and inseparably (Fig. 4b). Spongy parenchyma is randomly oriented (Fig. 4c). The proportion of mesophyll tissue in the highest $(81.4 \%)$ was Y6 site and lowest $(62.5 \%)$ in Y5 site. Stomata are presented below the

Table 2 The average dry weights of $P$. euphratica leaves at sites 1-6 of different sections

\begin{tabular}{|c|c|c|c|c|c|c|}
\hline \multirow[t]{2}{*}{ Section } & \multicolumn{6}{|c|}{ Average dry weight (g) } \\
\hline & 1 & 2 & 3 & 4 & 5 & 6 \\
\hline Yahepu & $0.7201^{\mathrm{a}}$ & $0.4697^{\mathrm{b}}$ & $0.4859^{\mathrm{c}}$ & $0.4762^{d}$ & - & - \\
\hline Yinsu & $0.6225^{\mathrm{a}}$ & $1.0247^{\mathrm{b}}$ & $0.7995^{\mathrm{c}}$ & $0.1367^{\mathrm{d}}$ & $0.5123^{\mathrm{e}}$ & $0.6351^{f}$ \\
\hline Abudali & $0.7588^{\mathrm{a}}$ & $0.5524^{\mathrm{b}}$ & $0.6204^{\mathrm{c}}$ & $0.4817^{\mathrm{d}}$ & $0.5505^{\mathrm{e}}$ & $0.6532^{\mathrm{f}}$ \\
\hline Kardayi & $0.7082^{\mathrm{a}}$ & $0.6332^{\mathrm{b}}$ & $0.5889^{\mathrm{c}}$ & $0.6454^{\mathrm{d}}$ & $0.6019^{\mathrm{e}}$ & $0.3258^{\mathrm{f}}$ \\
\hline
\end{tabular}

Note: For each row, the values with the same letter are not significantly different at 0.05 level.

Table 3 Water potential (WP) of $P$. euphratica leaves at different distances from Tarim River course in Yinsu section

\begin{tabular}{|c|c|c|c|c|c|c|}
\hline \multirow{2}{*}{ Distance (m) } & \multicolumn{3}{|c|}{ Water potential (bar) } & \multirow{2}{*}{ Range } & \multirow{2}{*}{ Standard deviation } & \multirow{2}{*}{ Coefficient of variation } \\
\hline & Max. & Min. & Mean & & & \\
\hline 100 & -15.00 & -23.67 & $-20.96 a$ & 8.67 & 4.94 & 16.69 \\
\hline 200 & -15.13 & -25.20 & $-22.13 \mathrm{ab}$ & 10.07 & 4.31 & 19.47 \\
\hline 300 & -18.00 & -30.00 & $-22.76 b$ & 12.00 & 4.94 & 21.69 \\
\hline
\end{tabular}

Note: The values are the mean for 5 samples. The values with different letters are significantly different at 0.05 level.

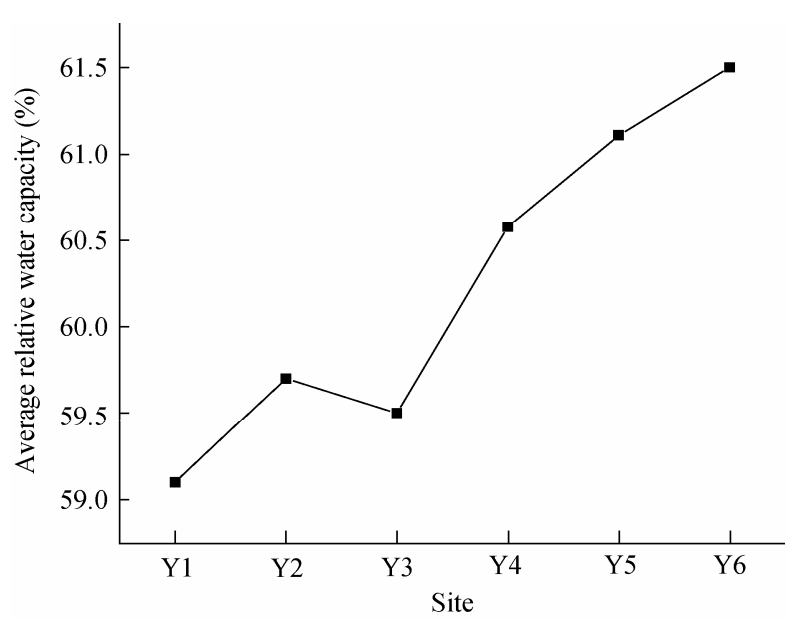

Fig. 3 Average relative water capacity of $P$. euphratica during the period of August, 2006-2008 in Yinsu section level of neighboring ordinary epidermal cells, and there are three layers of shield-like structures outside, sinking the stomata (Fig. 4d). The pentagonal or quadrilateral epidermal cells are smaller in the lower epidermis than that in the upper epidermis. The volume of the epidermal cells had decreased and the shape of the epidermal cells became irregular as the distance to the river increased. Stomata density was higher in the abaxial surface than that in the adaxial surface. The abaxial surface was always younger than the adaxial, which can be cleared by an ultrasonic wave. The hollow, hairy root structure cavity found in the leaf epidermis of Y6 site was connected to the epidermal cells (Fig. 4e). When the leaf catheter structures from different sampling sites were compared, all internal 

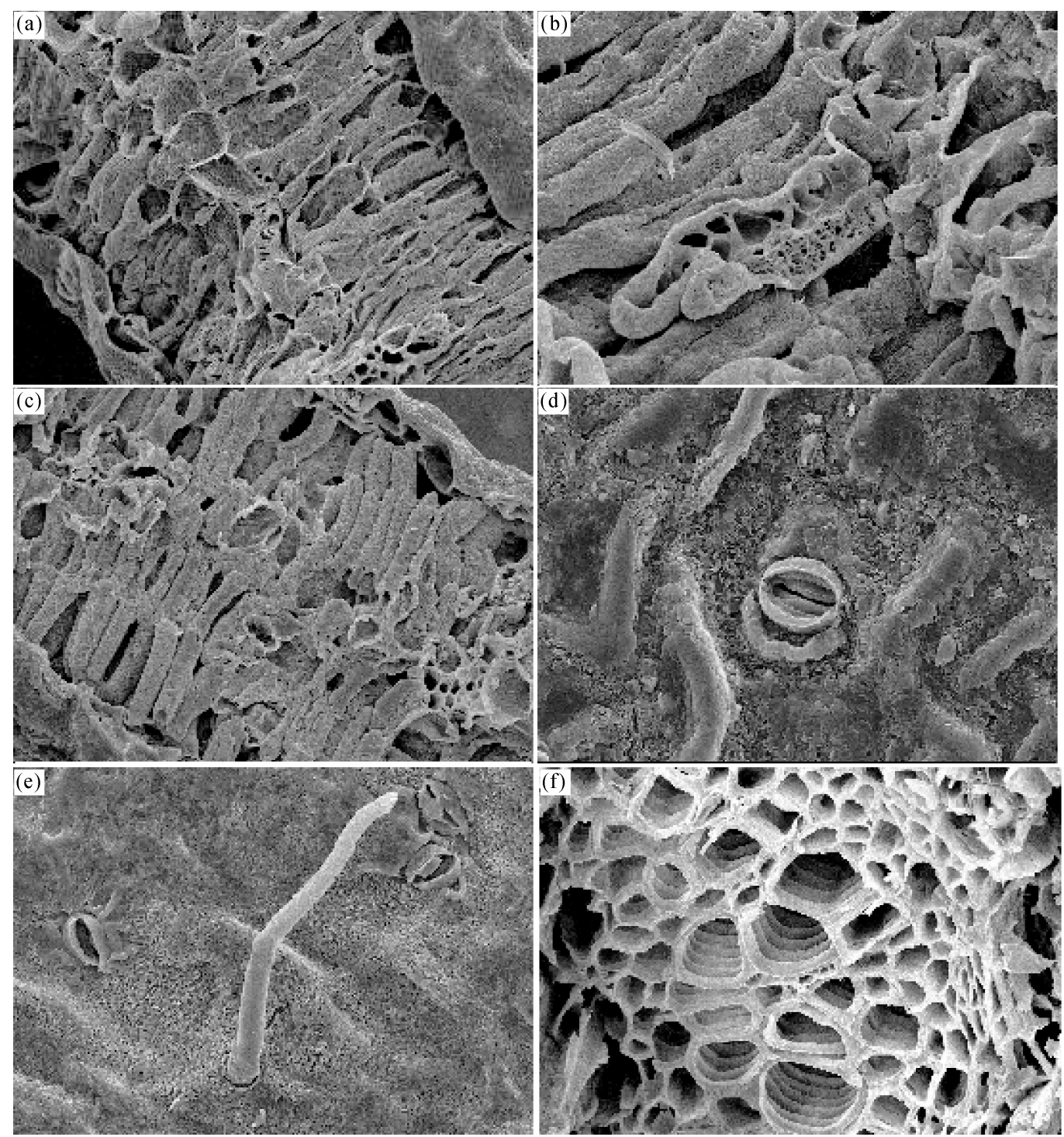

Fig. 4 Ultra-structure of $P$. euphratica leaves in Yinsu section

(a), (b) mean palisade tissue; (c) spongy parenchyma; (d) stomata; (e) long epidermal hair; and (f) spiral catheter

layers of the cylindrical catheter exhibited a spiral twist (Fig. 4f).

\section{Discussion and conclusion}

The results of the study confirmed that the leaves of $P$. euphratica in the different sections of Tarim River Basin show great variation. The lower reaches of Tarim River, located between the Taklamakan Desert and Kuluke Desert, lies in the lowest region of Tarim Basin, therefore, it becomes an ideal location for salt accumulation, due to the soluble salt taken by the surface and groundwater from the upper and middle reaches of Tarim River. Because the area is located in arid environment, the air temperature is much higher in the summer. The differences in humidity between day and night were significant, with a lower value during day and sometimes reaching saturation at night.

Since there is an exponential relationship between light absorption and the cumulative foliage area, the changes in the sizes of $P$. euphratica leaves can be considered functionally significant, related to the natural conditions where they developed. The upper wider ovate leaves, with thick cuticles, are useful for light absorption and reducing evaporation. The lower, young leaves are exposed to lower light, higher air humidity and are protected from wind. Thus, the leaf shape of $P$. euphratica changes gradually from a narrow lanceolate to a wider ovate upon maturation. The expansion of leaf diameter increases photosynthesis, 
which contributes to the production of some defensive metabolites, when subjected to stress. The phenomenon of survival of a small cluster of leaves, along with the stems, branches, and having enough flower and fruits, could be related to programming cell death under stress.

In the lower reaches of Tarim River, the scarcity of the soil water resources and the high evaporative demand of the atmosphere during the warmer seasons induce stress conditions. The survival of the plants in these adverse conditions requires long- and short-term plasticity responses and plants could develop stress avoidance mechanisms by changing its morphology and architecture (Xing, 2000), root development (Niinemets, 1996) or leaf anatomy (Long et al., 1994). When soil surface water becomes the limiting factor, trees have the potential to reach groundwater, and the adaptability of the root architecture may be an important determining factor of the competitive strength compared to foliar plasticity (Niinemets, 1996). However, the species was distributed at different sites, which was exposed to varying water tables. The various environments led to the leaf morpho-anatomical plasticity with respect to the combined conditions of soil salt, air temperature and humidity. One or all of these features could confirm the survival of the species, and enable them to compete with other species.

Bicolor is a common phenomenon among species that occupy shaded habitats; the leaf side facing away from the sun is lighter in color than the leaf surface facing the sun. The lighter surface may act as a reflective surface and enhance the participation of the spongy mesophyll in leaf photosynthesis (Maria, 2000). The abaxial surface of $P$. euphratica mature leaves is lighter than the adaxial surface because it is densely covered with hairs. Pubescence is another characteristic that confirms stress resistance (Ehleringer, 1980), because the presence of hairs on the aerial parts of the plants is regarded as an adaptation to arid conditions (Fahn, 1986) because the substantial increase in leaf reflectance and reduced radiation absorption which results in the reduction of leaf temperature. The long hair found in the site 6 of Yinsu section could be a special structure of epidermis that may be related to increased aridity. Water stored in hollow hairs could help to reduce the degree of injury with increasing stress due to drought.

The changes in the sizes, especially the shapes of the $P$. euphratica leaves, can be considered to have a functional significance related to the different natural conditions. The narrow, lanceolate leaves in the new branches help to reduce evaporation, while the wider, ovate leaves on the mature branches are capable of capturing as much light as possible, protecting them from the wind by thick cuticle and the presence of wax on them. In addition, self-orientation of the $P$. euphratica can decline leaf temperature and minimize water consumption. These characteristics may be related to the high solar irradiation of the region as increased light has a detrimental impact on the photosynthetic performance (Smith et al., 1997).

Smith et al. (1997) suggested that bicolor laminar leaves containing stomata only in the abaxial epidermis may have evolved as a result of selective pressure to enhance light capture, while avoiding the detrimental effect of exposure to sunlight and minimizing water-loss due to transpiration. In terms of $P$. euphratica, the difference in color between the mature leaf surfaces is marked and the stomata density of the abaxial surface is higher than that of the adaxial surface. The higher stomata density in the adaxial surface of $P$. euphratica may be a response to the more arid habitat. The proportionally large leaf size of mature leaves could mean an additional mechanical advantage under stressed conditions.

However, the plasticity of the leaves' shape and morphology is insufficient, and the anatomical features, such as the volume and organization of the mesophyll tissue, should be taken into consideration (Fahn et al., 1992). The abundance of plastids in the cytoplasm and amyloid in the plastids was observed, and the osmophilic particles were obviously displayed in the cytoplasm and in the posterior margin of the vacuole. The chloroplast is very important and unique plant organ which has the ability to convert light energy into chemical energy, and then store the energy as carbohydrates. The carbohydrates are mainly stored as starch granules in the chloroplasts. It can release enormous energy after hydrolysis and also provide carbon skeletons for protein synthesis at the same time (Hostak et al., 1986). These characteristics are defined as the structural mechanisms that increase photosynthesis per unit leaf area and enable greater water-use efficiency. The palisade tissue in the $P$. euphratica leaves is well developed and the palisade tissue cells are arranged perpendicular to the direction of the leaf epidermis cells and parallel to the direction of light. These characteristics can guarantee normal 
photosynthesis by leaves and also protect them from intense sunlight.

There are two epidermal cells in the mature leaves of the $P$. euphratica with a proportionally greater cuticle, which helps in reducing the diffusion of the water vapor from the leaf interior to the atmosphere. In addition, there is an obvious gap between the cuticle and the epidermal. This gap can play a role in storing water. On the other hand, it could lead to adaptation of leaves to the relatively large temperature ranges by isolating cell interior and exterior air. There are no significant relationships among the anatomical characteristics, such as stomata density and stomata opening, blade thickness, catheter lumen diameter and lumen wall thickness, epidermal thickness and palisade width, and the water table. However, the opening and density of the stomata of the abaxial surface was larger than those of the adaxial surface except blade thickness, palisade width and epidermal outer wall thickness. These characteristics indicate that water table is not the only inhibiting factor in the lower reaches of Tarim River and, therefore, the differences in the morphological and anatomical structures of $P$.

\section{References}

Charles A K, David D A. 2003. Evolution and plasticity of photosynthetic thermal tolerance, specific leaf area and leaf size: congeneric species from desert and coastal environments. New Phytologist, 160(2): 337-349.

Ehleringer J. 1980. Leaf morphology and reflectance in relation to water and temperature stress. New York: Wiley, 6: 295-308.

Fahn A. 1986. Structural and functional properties of trichomes of xeromorphic leaves. Annals of Botany, 57(5): 631-637.

Fahn A, Cutler D F. 1992. Xerophytes. Berlin: Gebruder Borntraeger, 180.

Gu R S, Jiang X N, Guo Z C. 1999. Structure characteristics associated with salt tolerance of Populus euphratica. Acta Botanica Sinica, 41(6): 576-579.

Hostak M S, Hienson C A, Duke S H. 1986. Starch-granule distribution between cell types of alfalfa nodules as affected by symbiotic development. Canada Journal of Botany, 65 (6): 1108-1115.

Leymarie J, Lasceve G, Vavasseur A. 1999. Elevated $\mathrm{CO}_{2}$ enhances stomatal responses to osmotic stress and abscisic acid in Arabidopsis thaliana. Plant, Cell and Environment, 22(3): 301-314.

Li X, Hou P, Yang P N. 2006. Analysis on the respond of the tree-rings of Populus euphratica to the change of moisture conditions in the lower reaches of the Tarim River. Arid Zone Research, 23(1): 26-31.

Long S P, Humphries S, Folkowski P G. 1994. Photoinhibition of photosynthesis in nature. Annual Review of Plant Physiology and Plant Molecular Biology, 45: 633-638.

Maria G K. 2000. Leaf variations in Elaeagnus angustifolia related to environmental heterogeneity. Environmental and Experimental euphratica could be related to other environmental factors.

Leaf variability in $P$. euphratica can be considered an adaptive advantage of the leaves in different habitats, marked by strong variations in solar radiation, air humidity, salinity, water table, temperature, and wind exposure. The leaves show many xeromorphic characters that enable the trees to maintain their normal development even under unfavorable conditions. These results confirmed the hypothesis that variations in the developmental responses of the $P$. euphratica leaves to spatial heterogeneity are related to its ecological strategies. This species relies, at least partially, on its foliar plasticity to overcome the environmental changes in different places, and even compete against other species in places where spatial environment heterogeneity can easily manifest, such as in a river valley of the lower reaches of Tarim River.

\section{Acknowledgements}

This research was funded by the National Natural Science Foundation of China (31060062 and 11014010) and the Shihezi University Program (Q9yy200814).

Botany, 44: 171-183.

Niinemets U. 1996. Changes in foliage distribution with relative irradiance and tree size: differences between the sapling of Acer platanoides and Quercus robur. Ecological Research, 11(3): 269-281.

Shin W, Katsumi K, Yuji I, et al. 2000. Effects of saline and osmotic stress on proline and sugar accumulation in Populus euphratica in vitro. Plant Cell, Tissue and Organ Culture, 63 (3): 199-206.

Smith W K, Vogelman T C, Delucia E H. 1997. Leaf form and photosynthesis. BioScience, 47(11): 785-793.

Wang F, Umut Halik, Conradie B, et al. 2009. Under the crown height of Populus euphratica along the Arghan section in the lower reaches of Tarim River in response to the emergency water transportation. Ecology and Environment, 18(1): 286-291.

Wang R H, You X X. 1999. The design and practice of Populus euphratica resources information management system. Entironmental Protection of Xinjiang, 21(4): 1-5.

Xing Y, Zhang D W, Gong X B. 2000. Comparative study on the morphology of Artemisla plants from northeastern China. Bulletin of Botanical Research, 20(1): 48-57.

Zhang J Q, Wang D X, Ma Q. 1996. Research on fire characteristics and ecological adaptability of populus wood in Tarim Basin. Journal of Northwest Forestry College, 11(1): 30-34.

Zhuang L, Li W H, Yuan F, et al. 2010. Ecological adaptation characteristics of Populus euphratica and Tamarix ramosissima leaf microstructures in the lower reaches of Tarim River. Acta Ecologica Sinica, 30(2): 62-66. 